\title{
Myxoid Dermatofibrosarcoma Protuberans
}

National Cancer Institute

\section{Source}

National Cancer Institute. Myxoid Dermatofibrosarcoma Protuberans. NCI Thesaurus.

Code C38106.

A morphologic variant of dermatofibrosarcoma protuberans characterized by the presence of extensive myxoid stroma formation and a storiform growth pattern. 